\title{
Selective Spatiotemporal Vulnerability of Central Nervous System Neurons to Pathologic TAR DNA-Binding Protein 43 in Aged Transgenic Mice
}

Annika van Hummel, ${ }^{*}$ Gabriella Chan, ${ }^{*}$ Julia van der Hoven, ${ }^{\dagger}$ Marco Morsch,${ }^{\ddagger}$ Stefania Ippati, ${ }^{\dagger}$ Lisa Suh, ${ }^{\dagger}$ Mian $\mathrm{Bi}^{\dagger}$

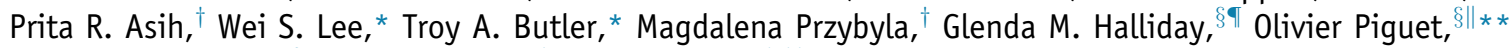
Matthew C. Kiernan, ${ }^{\S}$ Roger S. Chung, Lars M. Ittner, ${ }^{\dagger, \dagger}$ and Yazi D. Ke*

From the Motor Neuron Disease Unit* and the Dementia Research Unit ${ }^{\dagger}$ Department of Anatomy, School of Medical Sciences, Faculty of Medicine, University of New South Wales, Sydney; the Department of Biomedical Sciences, ${ }^{\ddagger}$ Faculty of Medicine and Health Sciences, Macquarie University Sydney, Sydney; the Brain and Mind Centre, ${ }^{\S}$ Central Clinical School, " and School of Psychology, "University of Sydney, Sydney; the ARC Centre of Excellence in Cognition and its Disorders, ** Sydney; and Neuroscience Research Australia, ${ }^{\dagger \dagger}$ Sydney, New South Wales, Australia

Accepted for publication March 8, 2018.

Address correspondence to Yazi D. Ke, Ph.D., Motor Neuron Disease Unit, Room 232, Level 2, Wallace Wurth Building, University of New South Wales, Sydney, NSW 2052, Australia. E-mail: yazi ke@unsw.edu.au.

\begin{abstract}
Amyotrophic lateral sclerosis (ALS) is a rapidly progressing and fatal disease characterized by muscular atrophy because of loss of upper and lower motor neurons. Histopathologically, most patients with ALS have abnormal cytoplasmic accumulation and aggregation of the nuclear RNA-regulating protein TAR DNA-binding protein 43 (TDP-43). Pathogenic mutations in the TARDBP gene that encode TDP-43 have been identified in familial ALS. We have previously reported transgenic mice with neuronal expression of human TDP-43 carrying the pathogenic A315T mutation (iTDP- $43^{A 315 T}$ mice), presenting with early-onset motor deficits in adolescent animals. Here, we analyzed aged $i T D P-43^{A 315 T}$ mice, focusing on the spatiotemporal profile and progression of neurodegeneration in upper and lower motor neurons. Magnetic resonance imaging and histologic analysis revealed a differential loss of upper motor neurons in a hierarchical order as $i T D P-43^{A 315 T}$ mice aged. Furthermore, we report progressive gait problems, profound motor deficits, and muscle atrophy in aged $i T D P-43^{A 315 T}$ mice. Despite these deficits and TDP-43 pathologic disorders in lower motor neurons, stereological analysis did not show cell loss in spinal cords. Taken together, neuronal populations in aging $i T D P-43^{A 315 T}$ mice show differential susceptibility to the expression of human TDP-43 ${ }^{\text {A315T }}$. (Am J Pathol 2018, 188: 1447-1456; https://doi.org/10.1016/j.ajpath.2018.03.002)
\end{abstract}

Amyotrophic lateral sclerosis (ALS) is a rapidly progressive and fatal neurodegenerative condition of the motor nervous system affecting frequently individuals in their prime. Although progressive muscle weakness and degeneration are the main symptoms of ALS, the disease shares symptoms, neuropathology, and genetics with frontotemporal lobar degeneration as part of a disease continuum. ${ }^{1}$ The neuropathologic hallmark shared by most patients with ALS and approximately one-half of the patients with frontotemporal lobar degeneration is ubiquitinated protein deposits in neurons that contain the TAR DNA-binding protein 43 (TDP43). ${ }^{2}$ Furthermore, TDP-43 inclusions have been described in a large proportion of patients with Alzheimer disease, further implicating its role in neurodegeneration. ${ }^{3}$
Under physiological conditions, TDP-43 localizes predominantly to the nucleus of cells, where it is involved in RNA processing. ${ }^{4}$ Nuclear localization and export sequences within the amino-terminal of TDP-43 mediate its

Supported by Australian National Health \& Medical Research Council (NHMRC) grants 1037746 (L.M.I., G.M.H., and M.C.K.), 1081916 (L.M.I.), 1132524 (L.M.I., G.M.H., M.C.K., and O.P.), 1143848 (Y.D.K.), 1095215 (R.S.C.), and 1143978 (Y.D.K.); Australian Research Council grants DP150104321 (Y.D.K.), DP170100781 (L.M.I.), and DP170100843 (L.M.I.); Motor Neurone Disease Australia grant GIA1824 (L.M.I.); the University of New South Wales; NHMRC R.D. Wright Biomedical Fellow grant 1123564 (Y.D.K.); NHMRC Senior Research Fellow grant 1103258 (O.P.); and NHMRC Principal Research Fellow grant 1136241 (L.M.I.).

Disclosures: None declared. 
subcellular localization. ${ }^{5}$ In disease, TDP-43 becomes increasingly phosphorylated, ubiquitinated, and accumulates in the cytoplasm of neurons, forming inclusions. ${ }^{2}$ Although the pathomechanisms associated with TDP-43 in ALS remain poorly understood, there is increasing evidence that soluble and fragmented species of TDP-43 contribute significantly to neuronal dysfunction and degeneration. ${ }^{6-8}$ Similarly, loss of physiological functions in the nucleus may contribute to disease onset and progression. ${ }^{9}$

In a small number of familial ALS families, mutations in the TDP-43-encoding gene TARDBP have been identified, ${ }^{10,11}$ further supporting an important role in disease. Furthermore, many other genes carrying mutations found in familial ALS are associated with TDP-43 pathologic disorders, including the most frequent ALS gene C9ORF72. ${ }^{12,13}$

Identification of TDP-43 as a major constituent of neuronal ubiquitinated inclusions in ALS and the identification of pathogenic and functional mutations in TARDBP have facilitated the generation of transgenic TDP-43 mouse models. ${ }^{14}$ Accordingly, both mutant and nonmutant TDP-43 have been expressed in mice by using different promoters $^{6,7,15-20}$ to model aspects of ALS in vivo. The phenotypic presentation varies between different TDP-43 transgenic lines from reproducing some neuropathologic features of ALS to early-onset functional deficits, significant neuronal cell loss, and reduced survival. Given the prevalence of TDP-43 pathologic disorders in ALS, transgenic TDP-43 mouse models have recently gained attention for testing of novel therapeutic approaches. ${ }^{21-24}$ Detailed characterization of phenotype progression in individual TDP-43 transgenic lines is required to facilitate further studies, including novel therapeutic interventions. ${ }^{25}$

We have previously reported early-onset motor and behavioral deficits in young transgenic mice expressing human TDP-43 with the pathogenic familial A315T mutation in central nervous system neurons $\left(i T D P-43^{A 315 T}\right) .{ }^{6}$ In this report, we extend our earlier findings of motor deficits and neuropathology to aged $i T D P-43^{A 315 T}$ mice, revealing selective vulnerability of central nervous system neurons to pathologic TDP-4 $3^{A 315 T}$ expression in vivo.

\section{Materials and Methods}

\section{Mice}

Transgenic mice with expression of neuronal transactivator [line mThy1.2-tTA(6)] and human A315T mutant TDP-43 under control of the tetracycline-responsive element promoter [line pTRE-TDP-43 $\left.3^{A 315 T}(13)\right]$ have been described previously. ${ }^{6,26}$ Mice were maintained on a C57B1/ 6 background and housed on a 12-hour light/dark cycle with access to standard chow and water ad libitum. Both male and female mice were used throughout this study. All experiments were approved by the University of New South Wales animal care and ethics committee.
MRI

Magnetic resonance imaging (MRI) was performed as described in detail before. ${ }^{6}$ All brains were mapped to the Australian Mouse Brain Mapping Consortium mouse brain atlas with the use of an affine registration (12 DOF). ${ }^{27}$ Brain atlas images were rotated, scaled, and sheared to match MRI scanned images. The motor and visual cortices were individually delineated through consecutive coronal images, using this transformed label map as a guide. The caudate putamen and cerebellum are visually distinct areas, and so segmentation was performed manually on these regions. Image registration was performed according to voxel data, which are independent of genotype of mice. All volumes were calculated and rendered in consecutive coronal MRI images with 3D Slicer software version 4.7.0 (NIH, Bethesda, MD; https://hpc.nih.gov/apps/3Dslicer.html).

\section{Gait Analysis}

Gait of mice was analyzed using a DigiGait system (Mouse Specifics Inc., Boston, MA). Briefly, individual mice ( $n=5$ to 14 per group) were placed in an enclosed chamber on a transparent, motorized treadmill belt and allowed to acclimatize to the machine at a speed of $6 \mathrm{~cm} / \mathrm{second}$. Mice were then recorded for at least four complete strides at a belt speed of 15 to $17 \mathrm{~cm} / \mathrm{second}$, with the paws captured by a camera from underneath. Gait analysis was performed in 1-month-old mice [ $n=10$ (non-tg), $n=28$ (single-tg), $n=10$ (iTDP-43)], 3-month-old mice [ $n=4$ (non-tg), $n=12$ (single-tg), $n=4$ (iTDP-43)], and 8-month-old mice [ $n=4$ (non-tg), $n=14$ (single-tg), $n=10$ (iTDP-43)]. A range of spatial and temporal gait parameters was calculated by DigiGait software (DigiGait Analyser version 14.5 and DigiGait Imager version 14; Mouse Specifics Inc.) for each limb to detect differences in gait between transgenic and nontransgenic mice across different ages.

\section{Motor Testing}

All motor tests were performed as previously described, ${ }^{6,28}$ with 7 (non-tg), 7 (single-tg), and 7 (iTDP-43) mice used.

\section{Rota-Rod}

Motor performance of mice was determined using a Rotarod (Ugo Basile, Varese, Italy) in acceleration mode (5 to $60 \mathrm{rpm}$ ) over 120 seconds. The longest time each mouse remained on the turning wheel of five attempts per session was recorded. Mice were previously recorded daily during 10 days and five trials per day, with no differences observed between mice trained longer or shorter on the Rotarod. As such, only the 3 days of testing were done for the rest of the study.

\section{Pole Test}

To test strength and coordination, mice were placed at the apex of a vertical pole $(47.5 \mathrm{~cm}$ length of dowel, diameter 
$0.8 \mathrm{~cm}$ ) facing upward. The time taken to turn around, descend the pole, and reach the ground (with all four paws) was measured with a maximum time of 120 seconds. Mice underwent one to two training sessions before the test session, during which the best time was taken of two trials. Mice who were unable to descend the pole (slipped or fell) were given the maximum time.

\section{Hanging Wire Test}

Mice were allowed to hang on an inverted rectangular wire mesh over a Perspex box for a maximum of 3 minutes, and latency to falling off was recorded (longest time of two attempts). ${ }^{29}$

\section{Grip Strength Test}

A grip strength meter (Ametek Chatillon, Berwyn, PA) was used to measure the force exerted by the forelimbs of the mouse. Mice were placed such that they had a double overhand grip on a thin metal wire attached to the meter and were pulled away from the meter in a horizontal direction until they let go, and a peak force $(\mathrm{N})$ was recorded at the moment when the mice let go. The highest force from five attempts was recorded.

\section{Histology and Staining}

For immunohistochemistry, tissues were embedded in paraffin and sectioned. Sectioned tissues were stained as previously described. ${ }^{30}$ The next 12-month-old mice were used for all histologic analysis $[n=9$ (non- and single tg) and $n=5$ (iTDP-43)]. Primary antibodies were against human TDP-43 (Proteintech, Chicago, IL), choline acetyltransferase (ChAT; Millipore, Burlington, MA), synaptophysin (Abcam, Cambridge, UK), and laminin (Sigma-Aldrich, St. Louis, MO). Sections were imaged on an Olympus BX51 microscope equipped with a DP70 camera (Olympus, Tokyo, Japan). Cortical thickness was determined on sagittal sections by using the measurement tool of the microscope software. Neuromuscular junction (NMJ) staining with bungarotoxin (SigmaAldrich) and synaptophysin antibody was done on fresh-frozen sections of tibialis anterior muscle as previously described. ${ }^{31}$

\section{Statistical Analysis}

Statistical analysis was performed with the Prism software package version 6.0 (GraphPad Inc., San Diego, CA) by using $t$-tests for pairwise comparison and analysis of variance for group comparison. All values are presented as means $\pm \mathrm{SEM}$.

\section{Results}

Loss of Upper Motor Neurons in Aged iTDP- $43^{A 315 T}$ Mice

Mutant TDP-43-expressing iTDP-43 ${ }^{A 315 T}$ mice were compared with single-transgenic [nonexpressing pTRE-
TDP- $\left.43^{\mathrm{A} 315 \mathrm{~T}}(13)\right]$ or nontransgenic control littermates throughout the study. We previously reported reduced brain and hippocampal volume in 6-month-old $i T D P-43^{A 315 T}$ mice compared with controls, without analyzing effects on other brain areas. ${ }^{6}$ To further analyze this MRI data set, volumetric analysis of different cortical brain areas was performed with brain atlas-guided mapping (Figure 1A). For comparison, corresponding tissue sections were immunohistochemically stained for transgenic $T D P-43^{A 315 T}$ expression pattern depicted at the same level (Figure 1A). Comparing volume loss with the overall size of the brain, only atrophy of cortical motor areas was significant in $i T D P-43^{A 315 T}$ mice compared with controls, whereas visual cortex, striatum, and cerebellum showed no relative atrophy (Figure 1B).

iTDP-43 ${ }^{\text {A315T }}$ mice had cortical atrophy and loss of transgenic $T D P-43^{A 315 T}$-expressing layer $\mathrm{V}$ neurons at 4.5 months of age. ${ }^{6}$ To determine progression of cortical atrophy, the analysis was extended to older mice. Brain area-specific analysis of immunostained brain sections showed that numbers of neurons expressing transgenic $T D P-43^{A 315 T}$ in the motor cortex decreased mostly by 6 months in $i T D P-43^{A 315 T}$ mice (Figure 1C). The continuing significant shrinkage of the motor cortex at 12 months of age, however, resulted in higher density of the remaining $i T D P-43^{A 315 T}$-expressing transgenic neurons. Accordingly, density of $T D P-43^{A 315 T}$ - positive neurons per area in layer II/III decreased from 2 to 6 months of age (number of neurons $/ 0.1 \mathrm{~mm}^{2}: 100.1 \pm 5.5$ versus $61.3 \pm 6.1 ; n=4$; $P=0.0033$ ), consistent with loss of neurons, but apparently increased from 6 to 12 months (number of neurons $/ 0.1 \mathrm{~mm}^{2}$ : $61.3 \pm 6.1$ versus $139.1 \pm 2.5 ; n=4 ; P<0.0001)$. However, numbers of TDP-43 ${ }^{A 315 T}$-positive neurons actually remained comparable between 6 and 12 months of age, when considering the reduced thickness in layer II/III because of atrophy (number of neurons $/ 0.1 \mathrm{~mm}^{2}: 49.0 \pm 4.9$ versus $57.9 \pm 1.1 ; n=4 ; P=0.1257$ ), suggesting no further loss of neurons after 6 months of age. Furthermore, ventricles were enlarged in 12-month-old iTDP-43 ${ }^{A 315 T}$ mice compared with littermate controls (data not shown). By 6 months of age, loss of TDP-43 $3^{A 315 T}$-expressing motor cortex neurons was apparent in cortical layers II/III and, in particular, V (Figure 1C). In contrast, the visual cortex of iTDP-43 ${ }^{\text {A315T }}$ mice was of comparable thickness at 2 and 6 months of age and showed only atrophy at 12 months of age with loss of TDP-43 $3^{A 315 T}$-expressing neurons (Figure 1C). Quantification of cortical thickness confirmed progressive atrophy of the motor cortex in $i T D P-43^{A 315 T}$ mice compared with controls as they aged, whereas atrophy occurred in the visual cortex only at 12 months of age (Figure 1D). Staining with a deep cortical layer V/VI marker, CTIP2, further confirmed a loss of neurons in these motor cortical layers (Figure 1E). Accordingly, layer width and number of CTIP2-positive neurons were reduced in $T T D P-43^{A 315 T}$ mice compared with controls at 12 months of age (Figure 1F). Taken together, aged $i T D P-43^{A 315 T}$ mice had brain atrophy 
A

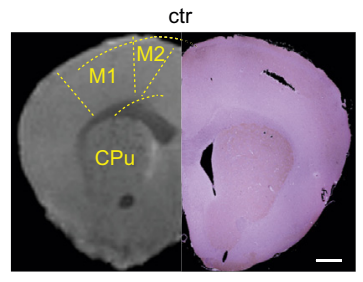

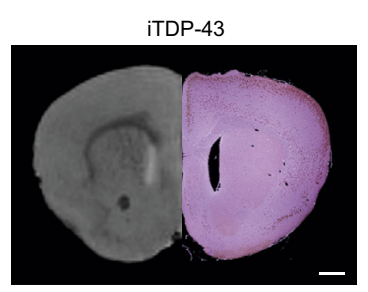

B

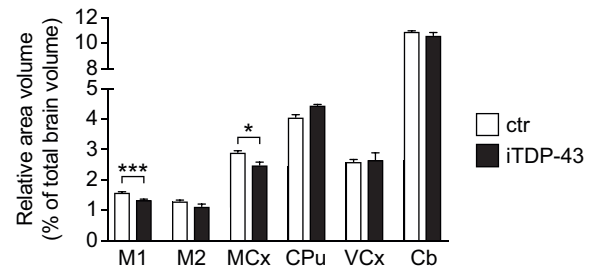

C
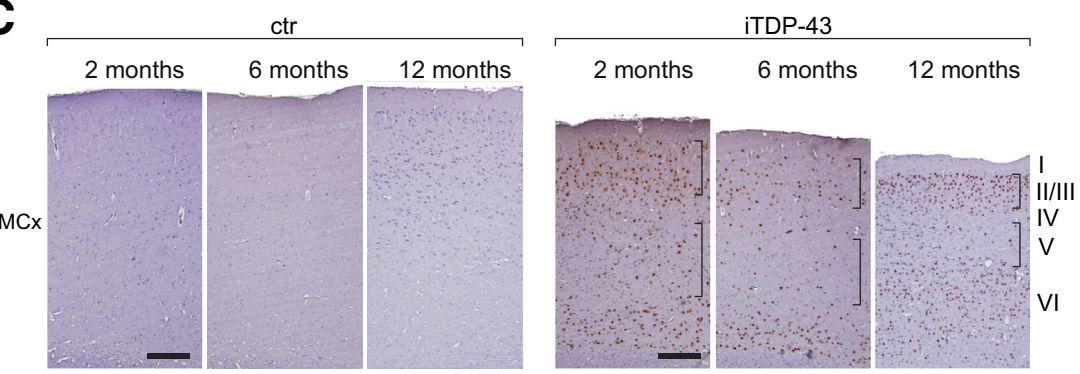

D
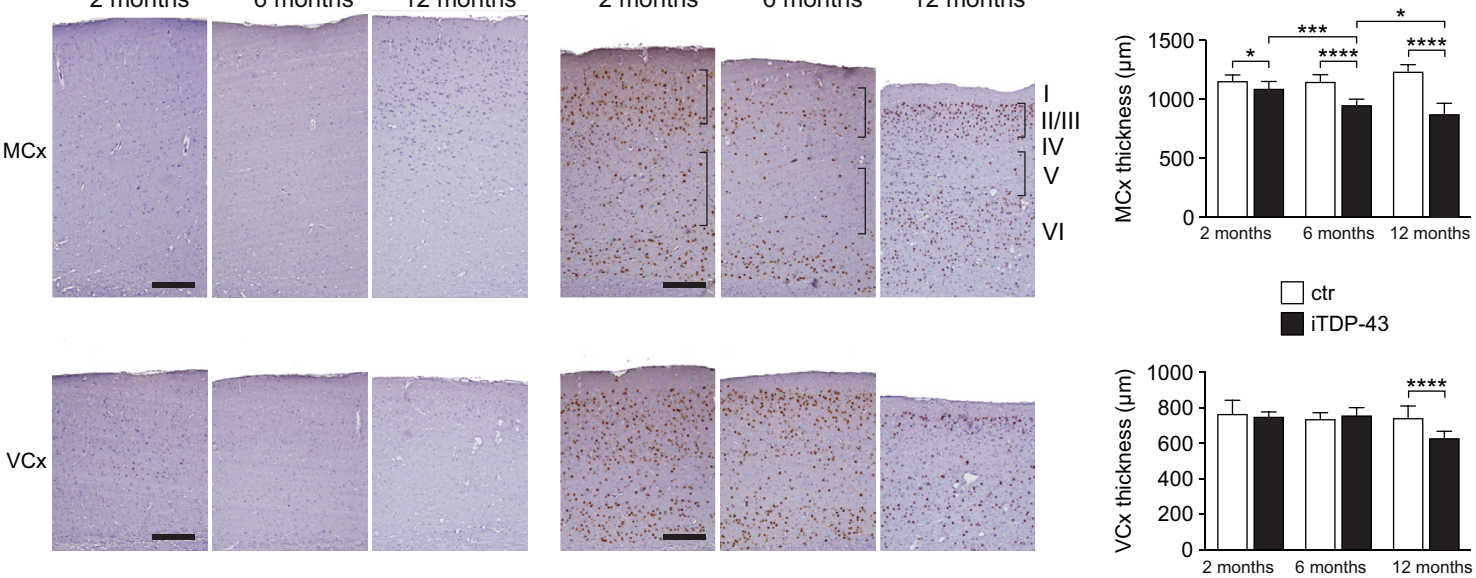

$\mathbf{E}$

ctr

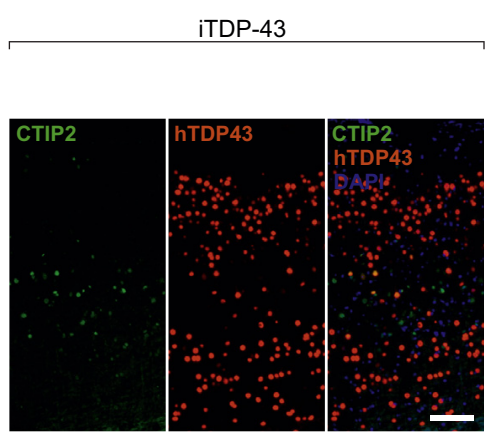

F

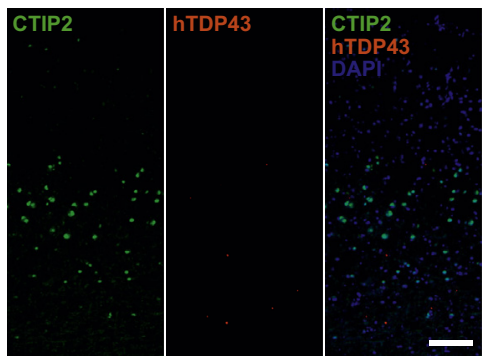

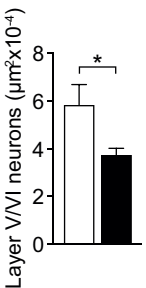

$\square \mathrm{ctr}$

Figure 1 Motor cortex atrophy in $i T D P-43^{A 315 T}$ mice. A: Representative magnetic resonance imaging (MRI) coronal sections of 6-month-old $i T D P-43^{A 315 T}$ (iTDP-43) and control (ctr) mice on the left. Brain areas are outlined. Representative staining for human TDP-43 (hTDP-43) in coronal sections of 6month-old iTDP-43 and ctr mice. B: Volumetric quantification of delineated brain areas showing reduced volumes in iTDP-43 mice compared with ctr mice. C: Motor cortex (MCx; top) and VCx (visual cortex; bottom) sagittal sections of iTDP-43 and ctr mice stained for transgenic hTDP-43 (brown). Neuronal layers II/III (top bracket) and V (bottom bracket) are indicated in iTDP-43 MCx sections. Note the progressive loss of transgenic hTDP-43-expressing cells in layers II/III/V and atrophy of MCx in iTDP-43 mice with no observed differences in layers I, IV, and VI. D: Progressive MCx thickness decrease in iTDP-43 mice compared with ctr mice over 2, 6, and 12 months of age. Comparable VCx thickness in iTDP-43 and ctr mice. E: Representative immunostaining of 12month-old iTDP-43 mice and ctr motor cortices for CTIP2 (green), a marker for deeper cortical layers V and VI, hTDP-43 (red), and nuclear DAPI (blue). F: Quantification showing reduced numbers of CTIP2-positive neurons and layer V/VI thickness in iTDP-43 mice compared with ctr mice. Data are expressed as means \pm SEM (B, D, and F). $n=3$ mice (B); $n=6$ mice (D). ${ }^{*} P<0.05,{ }^{* *} P<0.01,{ }^{*} * x<0.001$, and ${ }^{* * * *} P<0.0001$ ( $t$-test). Scale bars: $500 \mu \mathrm{m}$ (A); 200 $\mu \mathrm{m}(\mathbf{C}$ and $\mathbf{E})$. $\mathrm{Cb}$, cerebellum; $\mathrm{CPu}$, caudate putamen; $\mathrm{M1}$, primary motor cortex; $\mathrm{M}$, secondary motor cortex.

that particularly affected the motor cortex, first layer $\mathrm{V}$, followed by layer II/III neurons.

\section{Severe Motor Deficits in Aged iTDP-43 ${ }^{A 315 T}$ Mice}

Young $i T D P-43^{A 315 T}$ mice had early-onset motor deficits at 3 months of age. ${ }^{6}$ Here, 1-year-old $i T D P-43^{A 315 T}$ and control mice were subjected to the same motor test paradigms used earlier in adolescent mice. Specifically, the mice were tested using pole, wire, and beam tests as well as accelerating Rotarod. Notably, the aged $I T D P-43^{A 315 T}$ mice were not able to complete the beam tests, due to severe motor impairments (data not shown). Similarly, iTDP-43 ${ }^{A 315 T}$ mice were not capable of turning or descending when placed on the top of a vertical beam for pole testing, in contrast to control mice which reached the base within $27.9 \pm 15.5$ seconds (Figure 2A). Although 

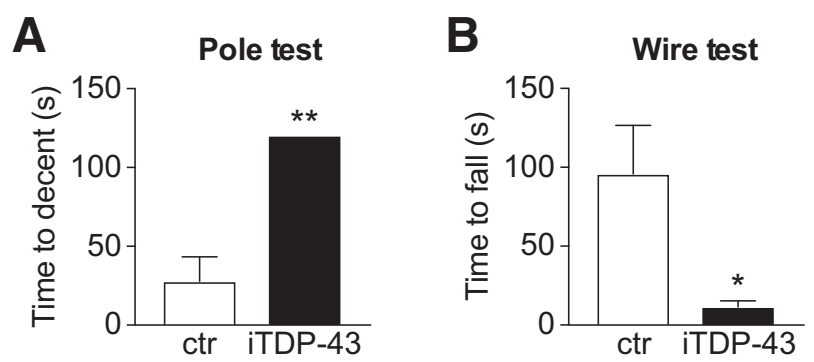

C

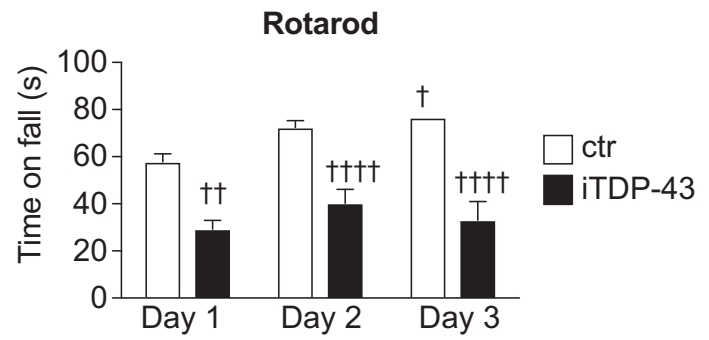

Figure 2 Severe motor deficits in aged $i T D P-43^{A 315 T}$ mice. A: Aged control (ctr) mice descend from the vertical pole significantly faster than iTDP-4 $3^{A 315 T}$ mice, which all fail to turn and descend within the test cutoff time of 120 seconds. B: iTDP- $43^{A 315 T}$ mice fall off an inverted wire mesh significantly earlier than ctr mice. C: $i T D P-43^{A 315 T}$ mice fall off the accelerating rod significantly earlier than ctr mice during Rotarod testing. Ctr mice but not $i T D P-43^{A 315 T}$ mice improve significantly over the testing period. Data are expressed as means \pm SEM. $n=7$ mice for each test. ${ }^{*} P<0.05,{ }^{*} P<0.01$ ( $t$-test) $;{ }^{\dagger} P<0.05,{ }^{\dagger \dagger} P<0.01$, and ${ }^{\dagger \dagger \dagger} P<0.0001$ (two-way analysis of variance; Sidak's post hoc test).

control mice were able to hold onto an inverted wire mesh for $95.9 \pm 30.7$ seconds, $i T D P-43^{A 315 T}$ mice dropped off significantly earlier at $11.4 \pm 3.9$ seconds (Figure 2B). During Rotarod testing, iTDP- $43^{A 315 T}$ mice failed to stay on the accelerating rod for more than $34.1 \pm 3.2$ seconds, whereas control animals remained on the rod for significantly longer at $68.8 \pm 5.7$ seconds (Figure 2C). Notably, control mice improved their performance on the Rotarod significantly over the three testing days, whereas $i T D P-43^{A 315 T}$ mice showed no improvement. Despite the severe motor deficits, limb paralysis or decreased survival was not observed in the colony of $i T D P$ $43^{A 315 T}$ mice, followed up to 1 year of age. Taken together, aged $i T D P-43^{A 315 T}$ mice had severe motor deficits.

\section{Progressive Gait Problems in Aging iTDP-43 ${ }^{A 315 T}$ Mice}

ALS is characterized by hypokinesia and gait deficits, ${ }^{32}$ and gait changes have not been previously investigated in iTDP- $43^{A 315 T}$ mice. Progressive muscle atrophy and motor problems observed in $i T D P-43^{A 315 T}$ mice would likely affect the overall movement of the animals. Therefore, $i T D P-43^{A 315 T}$ and control mice at different ages were subjected to digital gait analysis while moving on a treadmill, and different parameters of the gait dynamics and patterns were analyzed. At 8 months of age, the gait pattern of $i T D P-43^{A 315 T}$ mice was already different from nontransgenic and single transgenic littermate controls, with reduced step length and increased step frequency at the same treadmill speed (Figure 3, A and B). Digital analysis of individual stride phases revealed significantly reduced stride time (time to complete a full step cycle), stride length (distance covered per step), swing time (time paws are in the air during each step), and propel time (time spent to move forward), together with increased compensatory stride frequency in $i T D P-43^{A 315 T}$ mice compared with control mice at 8 months of age (Figure 3B). When comparing gait patterns over time, it was found that at 1 month of age, swing time of both front and hind limbs were already significantly reduced in $i T D P-43^{A 315 T}$ mice (Figure 3C). Similar deficits were detected at 3 and 8 months of age in $i T D P-43^{A 315 T}$ mice, with the relative deficits for the front limbs becoming larger over time (Figure 3C). Gait symmetry (ratio of fore limb stepping forward to hind limb stepping frequency) typically declines with age, as seen in nontransgenic and single transgenic controls, when comparing 1-month-old mice with 8-month-old mice (Figure 3D). Failure to decline in 8-month-old $i T D P-43^{A 315 T}$ mice indicated increased stepping frequency of the hind limbs to propel forward. At 1 month of age, paw areas at peak stance were unchanged in $i T D P-43^{A 315 T}$ mice (Figure $3 \mathrm{E}$ ). However, at 3 months of age paw areas at peak stance of the hind limbs were significantly smaller in $i T D P-43^{A 315 T}$ mice, and eventually both hind and front limbs were significantly affected at 8 months of age, indicative of progressive mechanical changes (Figure 3E). This was further supported by a reduced change of paw area over time in the hind limbs of 3-and 8-month-old iTDP-43 ${ }^{A 315 T}$ mice but not in 1-month-old iTDP-43 $3^{A 315 T}$ mice, suggesting problems with shifting load on and off the paws during walking (Figure $3 \mathrm{~F}$ ). Taken together, $i T D P-43^{A 315 T}$ mice displayed significant and progressive gait deficits as they aged.

\section{Muscle Atrophy in Aged iTDP-43 ${ }^{A 315 T}$ Mice}

Motor deficits in adolescent $i T D P-43^{A 315 T}$ mice were accompanied by muscle atrophy. ${ }^{6}$ With the use of cross sections of the tibialis anterior muscle, fiber diameter distribution was determined in 1-year-old $i T D P-43^{A 315 T}$ and control mice. Immunostaining of basal membrane laminin showed smaller muscle fibers in $i T D P-43^{A 315 T}$ mice compared with large diameter fibers in control muscles (Figure 4A). Muscle fiber diameter distribution in $i T D P$ $43^{A 315 T}$ mice was left shifted compared with control mice, in line with atrophy (Figure 4B). Consistent with muscle atrophy, forearm grip strength was significantly reduced in aged $i T D P-43^{A 315 T}$ mice compared with control mice (Figure 4C). Muscles from 5- and 8-month-old iTDP- $43^{A 315 T}$ mice and controls were stained with bungarotoxin to label postsynaptic acetylcholine receptors and antibodies to presynaptic synaptophysin to visualize the architecture of NMJs. Despite the apparent atrophy of muscles in $i T D P-43^{A 315 T}$ mice, NMJs presented similarly in control and $i T D P-43^{A 315 T}$ tibialis anterior muscles at both ages (Figure 4D). Accordingly, postsynaptic acetylcholine receptor distribution was indistinguishable between 

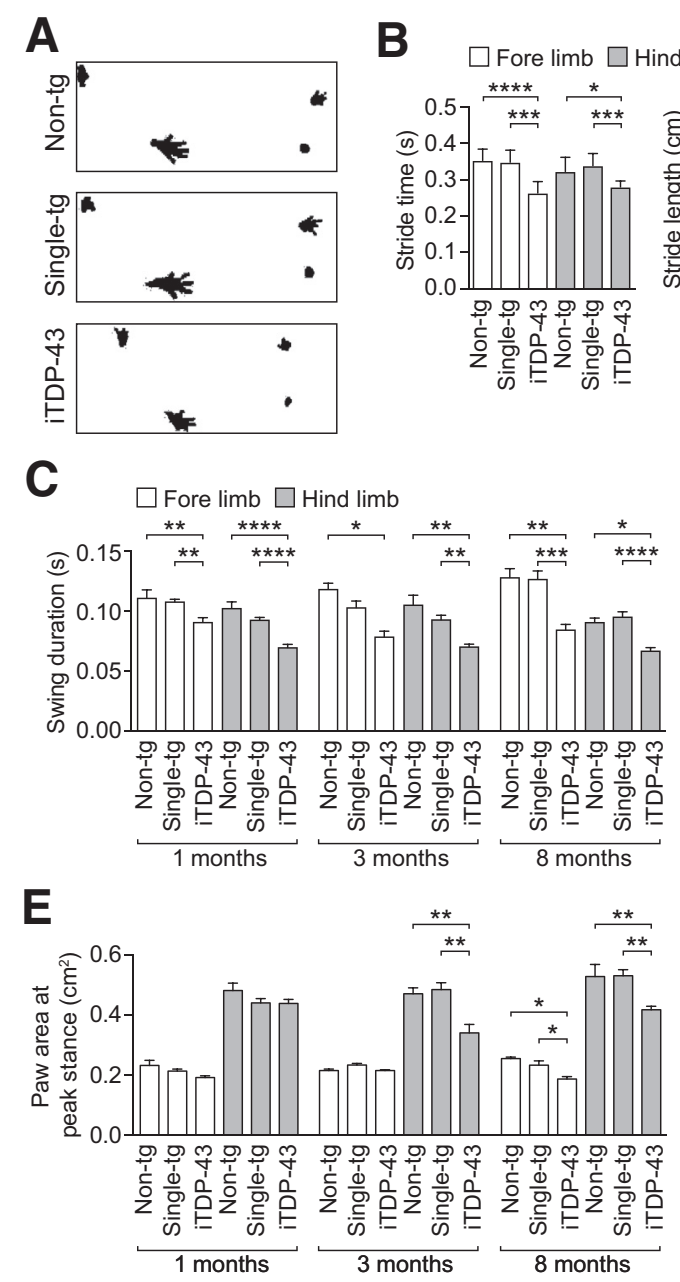

B $\square$ Fore limb $\square$ Hind limb
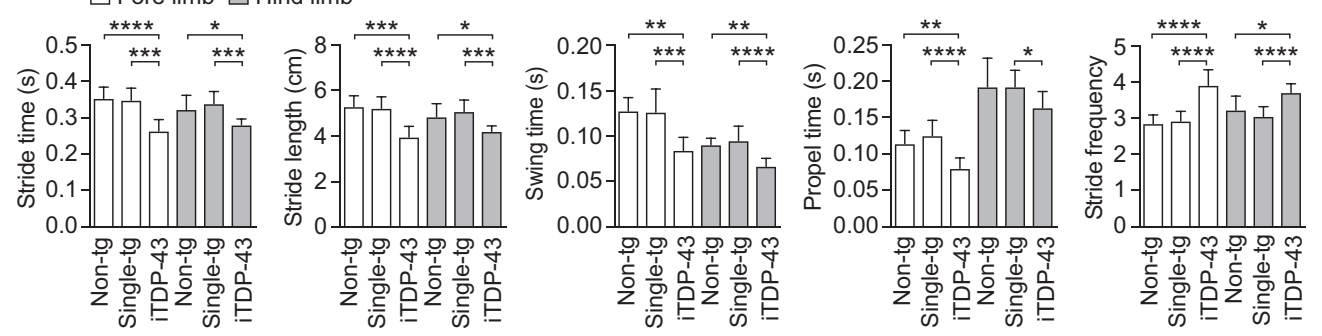

D
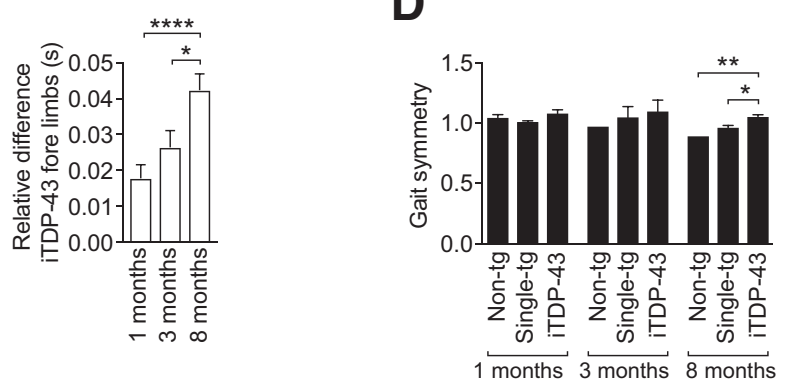

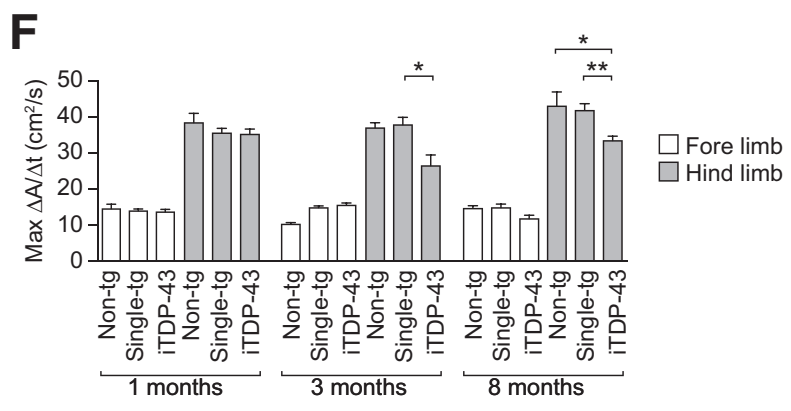

Figure 3 Progressive gait problems in aging $i T D P-43^{A 315 T}$ mice. A: Representative gait pattern of nontransgenic (non-tg) and single-transgenic (single-tg) control and $i T D P-43^{A 315 T}$ (iTDP-43) mice at 8 months of age. B: Gait parameters of non-tg, single-tg, and iTDP-43 ${ }^{A 315 T}$ mice. C Left: Reduced swing duration of fore and hind limbs in $i T D P-43^{A 315 T}$ mice compared with non-tg and single-tg controls. Right: Progressive decline in swing duration of fore limbs in $i T D P$ $43^{A 315 T}$ mice. D: Gait symmetry in non- $\mathrm{tg}$, single-tg, and $i T D P-43^{A 315 T}$ mice. E: Progressive decline in peak stance area of hind limbs followed by fore limbs in iTDP- $43^{A 315 T}$ mice compared with non-tg and single-tg controls. F: Reduced change of paw area in hind limbs of $i T D P-43^{A 315 T}$ mice compared with non-tg and single-tg controls. Data are expressed as means \pm SEM (B-F). $n=4$ non-tg mice (B); $n=14$ single-tg mice (B); $n=10$ iTDP-43 mice (B); $n=10$ non-tg 1month-old mice (C, left, and $\mathbf{D}-\mathbf{F}) ; n=28$ single-tg 1-month-old mice (C, left, and $\mathbf{D}-\mathbf{F}) ; n=10$ iTDP-43 1-month-old mice (C, left, and $\mathbf{D}-\mathbf{F}) ; n=4$ non-tg 3-month-old mice (C, left, and $\mathbf{D}-\mathbf{F}) ; n=12$ single-tg 3-month-old mice (C, left, and $\mathbf{D}-\mathbf{F}) ; n=4$ iTDP-43 3-month-old mice (C, left, and $\mathbf{D}-\mathbf{F})$; $n=4$ non-tg 8-month-old mice (C, left, and $\mathbf{D}-\mathbf{F}) ; n=14$ single-tg 8-month-old mice (C, left, and $\mathbf{D}-\mathbf{F}) ; n=10$ iTDP-43 8-month-old mice (C, left, and D-F); $n=10$ mice 1 month old (C, right), $n=4$ mice 3 months old (C, right); $n=10$ mice 8 months old (C, right). ${ }^{*} P<0.05 \mathrm{l},{ }^{* *} P<0.01,{ }^{* * *} P<0.001$, and ${ }^{* * * *} P<0.0001$ (one-way analysis of variance; Sidak's post hoc test).

$i T D P-43^{A 315 T}$ and control muscles at 5 and 8 months of age. Although presynaptic synaptophysin distribution was comparable in $i T D P-43^{A 315 T}$ and control muscles at 5 months of age, there was a nonsignificant trend to reduced synaptophysin staining in $i T D P-43^{A 315 T}$ mice at 8 months of age (Figure $4 \mathrm{E}$ ). Taken together, aged $i T D P-43^{A 315 T}$ mice had severe muscle atrophy, reduced grip strength, yet no overt changes to NMJs.

\section{TDP-43 Pathologic Disorders in Spinal Cord Motor Neurons of Aged iTDP-43 ${ }^{A 315 T}$ Mice}

Transgenic TDP-43 $3^{A 315 T}$ expression in spinal cord motor neurons was associated with TDP-43 pathologic disorders in adolescent $i T D P-43^{A 315 T}$ mice. ${ }^{6}$ To determine the degree of pathologic disorder in aged $i T D P-43^{A 315 T}$ mice, serial sections were stained at different levels of transgenic and control spinal cords with antibodies to human TDP-43 (Figure 5A). At all levels of $i T D P-43^{A 315 T}$ spinal cords, some large caliper motor neurons expressed transgenic $T D P-43^{A 315 T}$ with nuclear and abundant cytoplasmic localization. Serial sections stained for the motor neuron marker ChAT and human TDP-43 confirmed the small number motor neurons expressing TDP-43 $3^{A 315 T}$ (Figure 5B). Quantification of these stainings revealed comparable numbers of ChAT-positive motor neurons in both control and $i T D P-43^{A 315 T}$ mice (Figure 5B). Numbers of human TDP-43-positive neurons in spinal cords 

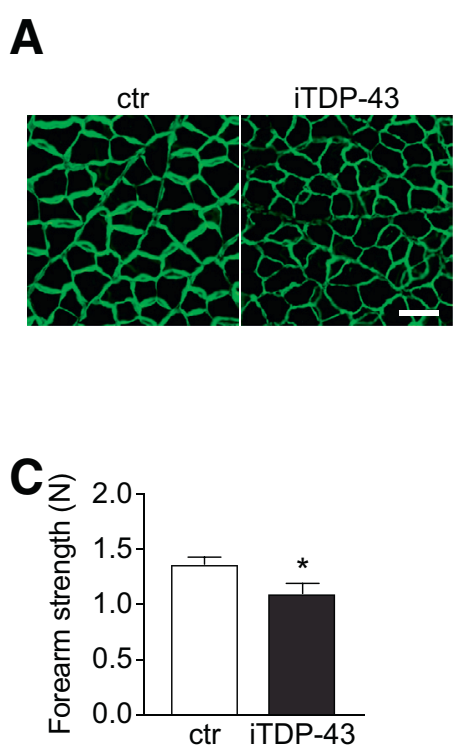

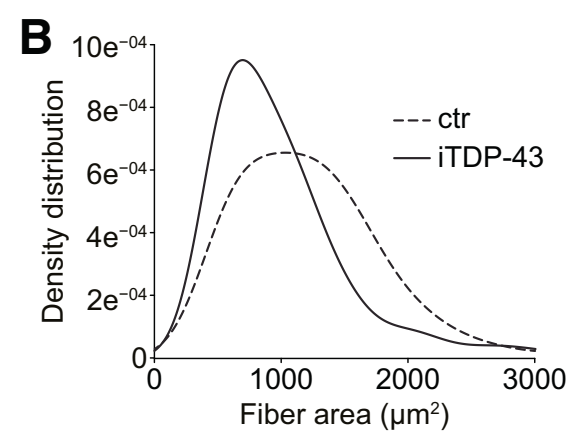

D
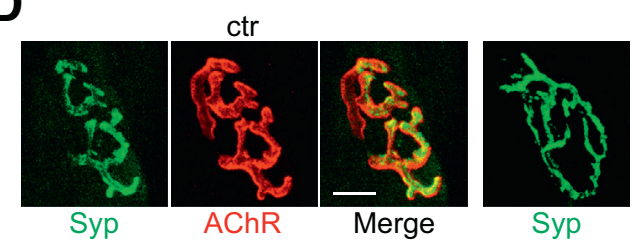

iTDP-43

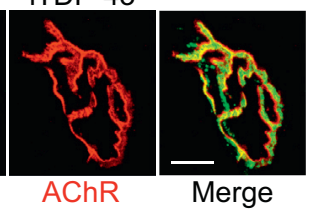

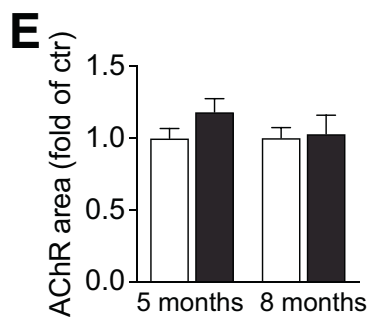
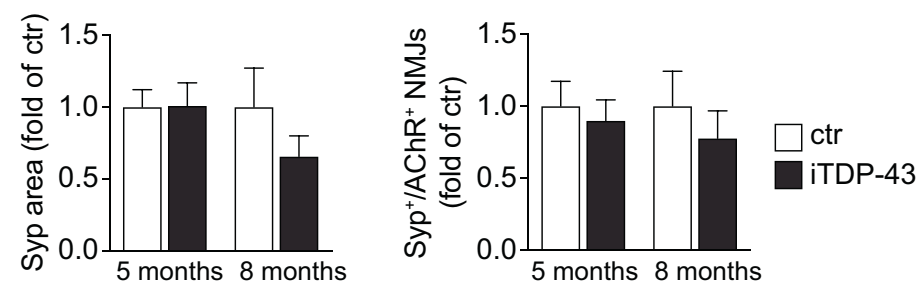

Figure 4 Substantial muscle atrophy in aged $i T D P-43^{A 315 T}$ mice. A: Staining of basal membrane laminin (green) of muscle sections from 12-month-old control (ctr) and iTDP-43 ${ }^{A 315 T}$ (iTDP-43) mice. B: Shifted muscle fiber diameter distribution in 12-month-old iTDP-43 mice compared with ctr mice. Reduced mean muscle fiber diameter in 12-month-old iTDP-43 mice compared with ctr mice. C: Reduced grip strength in 12-month-old iTDP-43 mice compared with ctr mice. D: Examples of postsynaptic acetylcholine receptor (AChR; red) and presynaptic synaptophysin (Syp; green) staining of neuromuscular junctions (NMJs) from 5- and 8-month-old ctr and iTDP-43 mice. E: Unchanged postsynaptic AChR and presynaptic Syp distribution and co-localization in NMJs of ctr and iTDP-43 mice. Data are expressed as means \pm SEM (B, C, E). $n=7 \mathrm{ctr}$ mice (B and C); $n=5$ iTDP-43 mice (B); $n=7$ iTDP-43 mice (C); $n=6 \mathrm{ctr}$ mice 5 months old $(\mathbf{E}) ; n=4$ iTDP-43 mice 5 months old $(\mathbf{E}) ; n=5$ ctr mice 8 months old $(\mathbf{E}) ; n=5$ iTDP-43 mice 8 months old (E). ${ }^{*} P<0.05$ (t-test). Scale bars: $50 \mu \mathrm{m}$ (A); $10 \mu \mathrm{m}$ (D).

of $i T D P-43^{A 315 T}$ mice were of similar numbers to that of ChAT-positive cells; however, only $14.1 \% \pm 4.1 \%$ of ChAT-positive motor neurons expressed transgenic human TDP-43, suggesting most of the human TDP-43-positive neurons were ChAT-negative. In addition to motor neurons, a large number of smaller cells also stained positively for transgenic $T D P-43^{A 315 T}$, with some cytoplasmic localization. Double-labeling with the neuronal marker NeuN confirmed purely neuronal expression of transgenic TDP$43^{A 315 T}$ throughout the gray matter of $i T D P-43^{A 315 T}$ spinal cords (Figure 5C). Co-staining with parvalbumin showed a near complete overlap with $T D P-43^{A 315 T}$, consistent with transgene expression in interneurons (Figure 5D). Astrocytes and microglia did not express TDP-4 $3^{A 315 T}$ (data not shown), and no staining of human TDP-43 was observed in control spinal cords. Taken together, transgenic TDP$43^{A 315 T}$ was expressed mostly in interneurons and some motor neurons in the spinal cord of $i T D P-43^{A 315 T}$ mice, where it localized to both the nucleus and cytoplasm, but did not cause overt lower motor neuron loss.

\section{Discussion}

In the present study, the phenotypic presentation of $i T D P$ $43^{A 315 T}$ mice was followed with age. At 1 year of age, iTDP- $43^{A 315 T}$ mice showed severe motor deficits, muscle atrophy, and cytoplasmic TDP-43 accumulation in spinal cord motor neurons. Furthermore, iTDP-43 ${ }^{A 315 T}$ mice developed progressive gait problems with aging.

We have previously reported a selective and progressive loss of pyramidal layer $\mathrm{V}$ neurons in the cortex of young $i T D P-43^{A 315 T}$ mice, with no change in layer II/III and VI neurons, despite marked expression of transgenic human TDP- $43{ }^{6}$ Similarly, there was no loss of spinal cord motor neurons in young $i T D P-43^{A 315 T}$ mice, but there was a muscle weakness and atrophy, indicating neuronal dysfunction. ${ }^{6}$ Cortical motor areas were relatively earlier affected by atrophy than other cortical areas in aged $i T D P$ $43^{A 315 T}$ mice, whereas there was still no overt loss of spinal cord motor neurons. Interestingly, layer II/III showed a significant degeneration in 12 -month-old $i T D P-43^{A 315 T}$ 
A

A

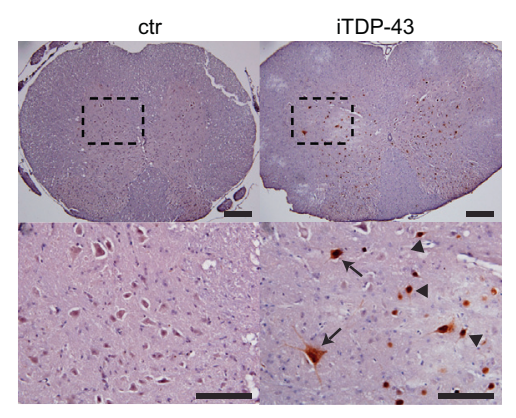

B
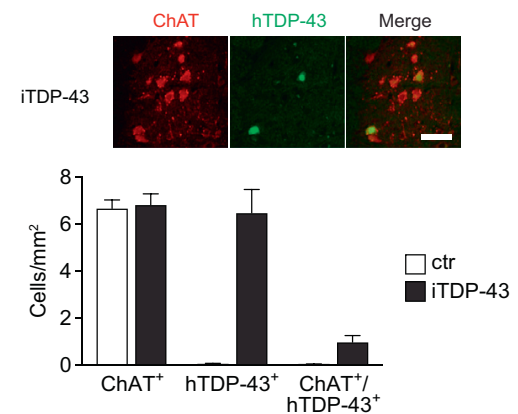

C

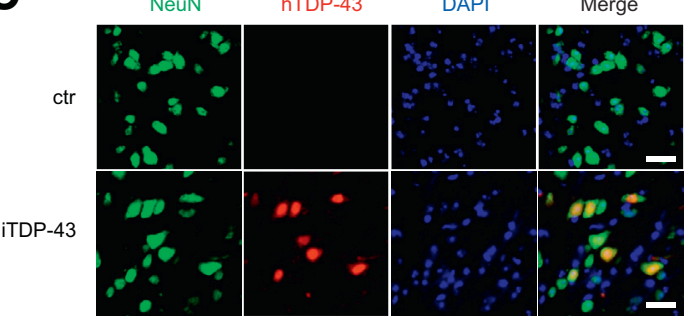

D

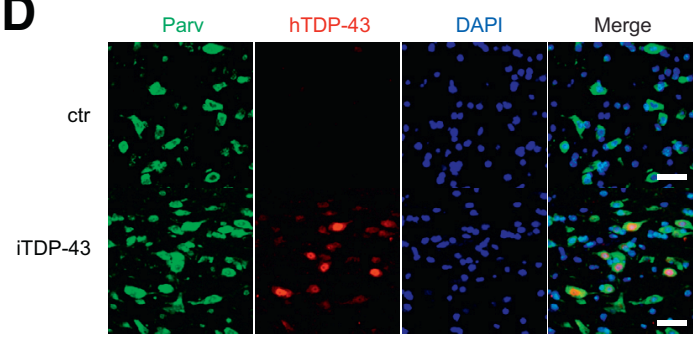

Figure 5 TDP-43 expression in spinal cord neurons in aged iTDP-43 ${ }^{A 315 T}$ mice. A: Representative sections of spinal cords from 12-month-old control (ctr) and $i T D P-43^{A 315 T}$ (iTDP-43) mice stained with human TDP-43 (hTDP-43)-specific antibodies (brown). Boxed areas in top panels indicate area shown at higher magnification in lower panels. Some large caliper motor neurons present with abundant cytoplasmic hTDP-43 staining (arrows), as well as several smaller neurons with hTDP-43 expression (arrowheads). B: Representative immunofluorescence images showing co-localization of transgenic TDP-43 ${ }^{A 315 T}$ and the motor neuron marker choline acetyltransferase (ChAT) in the spinal cords of iTDP-43 mice. Quantification of numbers of Chat-, hTDP-43-, and double-positive motor neurons of serial spinal cord section from ctr and iTDP-43 mice. C: hTDP-43-positive cells in the spinal cord of iTDP-43 mice are also NeuN-positive. D: hTDP-43-positive cells in the spinal cord of iTDP-43 mice are also positive for the interneuron marker parvalbumin (parv). Data are expressed as means \pm SEM (B). $n=10 \mathrm{ctr}$ mice (B); $n=5$ iTDP-43 mice (B). Scale bars: $200 \mu \mathrm{m}$ (A, top); $100 \mu \mathrm{m}$ (A, bottom); $50 \mu \mathrm{m}$ (B-D).

mice. In contrast, in the visual cortex only layer $\mathrm{V}$ neurons were affected at 12 months of age in $i T D P-43^{A 315 T}$ mice. Distinct effects of transgenic mutant TDP-43 expression on cortical neurons have also recently been reported in independent $T D P-43^{Q 331 K}$ and $T D P-43^{A 315 T}$ mouse strains. ${ }^{33,34}$ Furthermore, selective neuronal loss associated with TDP-43 pathologic disorders has recently been reported for cranial nerve motor nuclei, with cell loss in the hypoglossal nucleus 8 weeks after expression of a human TDP-43 variant that is excluded from entering the nucleus $(T D P-43-\triangle N L S)$ in aged mice, while sparing oculomotor, trigeminal, and facial nuclei. ${ }^{35}$ Expression of TDP-43 ${ }^{A 315 T}$ predominantly in parvalbumin-positive spinal cord interneurons and some motor neurons may impair neuronal circuits that control muscle function, thereby contributing to muscle atrophy in aged $I T D P-43^{A 315 T}$ mice in the absence of motor neuron loss (up to an age of 12 months analyzed here) or changes in NMJs. Lack of corticospinal afferents, due to upper motor neuron loss, may further contribute to the muscular dysfunction. However, the exact mechanism(s) underlying muscle atrophy in $i T D P-43^{A 315 T}$ mice remains to be shown. Taken together, the differential vulnerability of different neuron types may suggest the intriguing possibility that there are different pathologic TDP-43 species with distinct effects on neurons, and models such as the $i T D P-43^{A 315 T}$ mice may assist in future identification of these species.
Degeneration and TDP-43 pathologic disorders in motor areas and muscle disorders affected functional performance of $i T D P-43^{A 315 T}$ mice. Accordingly, motor testing of aged $i T D P-43^{A 315 T}$ mice indicated severe deficits, including the inability to perform tasks that young transgenic mice were once able to successfully complete. These findings are in line with our initial report of $i T D P-43^{A 315 T}$ mice with progression of motor deficits from none at 1 month of age to significant deficits already at 3 months of age. ${ }^{6}$ Therefore, iTDP $-43^{A 315 T}$ mice develop significant motor deficits early on, which persist as they age. This parallels the early degeneration of upper motor neuron areas and the progressive development of TDP-43 pathologic disorders in lower motor neurons and muscle wasting.

Patients diagnosed with ALS have been reported to have abnormal gait parameters. ${ }^{36-38}$ Digital and longitudinal gait analysis has not been done in TDP-43 transgenic ALS mouse models before. $i T D P-43^{A 315 T}$ mice have early-onset changes in gait parameters, which become more complex over time, including some deficits that progress from initially affecting hind limbs at 3 months of age to eventually impairing both fore and hind limbs at 8 months of age. Homozygous $T D P-43^{M 337 V}$ mice displayed irregular gait patterns at weaning together with a premature mortality, which precluded a detailed gait analysis over time. ${ }^{39}$ Furthermore, abnormal gait has been reported in several mouse models of ALS without transgenic TDP-43 
expression. Mancuso et $\mathrm{al}^{40}$ provided a detailed gait analysis of SOD ${ }^{\mathrm{G} 93 \mathrm{~A}}$ - expressing ALS mice before and after onset of overt motor deficits on the Rotarod. Similar to $i T D P$ $43^{A 315 T}$ mice, SOD1 ${ }^{G 93 A}$ mice developed progressively increasing gait deficits. Interestingly, analysis of the stride phases showed progressive increase in stride, stance, swing, and propulsion times and reduced stride frequency, ${ }^{40}$ as opposed to increased stride frequency and reduced stride phase times found in $i T D P-43^{A 315 T}$ mice. These differences may be explained because $S O D 1^{G 93 A}$ mice have a rapid progressive hind limb paralysis and loss of spinal cord motor neurons, whereas the $i T D P-43^{A 315 T}$ mice are characterized by slow progressive muscle atrophy, which limits limb strength and movement, and neuronal loss limited cortical motor areas, but do not develop paralysis. Furthermore, transgenic TDP- $43^{A 315 T}$ expression was found predominantly in spinal cord interneurons and only a limited number of motor neurons, which may suggest that dysfunction of neuronal circuits that controls rhythm of locomotor movement, including left-right alternation and flexor-extensor equilibrium, ${ }^{41}$ rather than loss of spinal cord motor neurons contribute to gait problems in $i T D P$ $43^{A 315 T}$ mice. However, Vergouts et $\mathrm{al}^{42}$ found early gait anomalies with reduced stride length in SOD $1{ }^{\mathrm{G} 93 \mathrm{~A}}$ mice, ${ }^{42}$ comparable with $i T D P-43^{A 315 T}$ mice. Furthermore, a polyGA transgenic C9ORF72 model developed progressive gait anomalies and TDP-43 pathologic disorders. ${ }^{43}$ Taken together, abnormal gait is found in several ALS mouse models; however, its presentation depends on the underlying phenotype.

\section{Conclusions}

The present study showed the progression of functional deficits in $i T D P-43^{A 315 T}$ mice as they age, including a detailed analysis of progressive gait problems. Furthermore, differences in neuronal loss between cortical motor areas and spinal cord provided additional evidence to the concept of selective vulnerability of different neuronal populations to TDP-43 pathologic disorders, ${ }^{6,35}$ and they may assist in future identification of specific toxic TDP-43 species. Finally, differences in phenotype between ALS mouse models, including between TDP-43 transgenic stains, highlight the importance of a thorough characterization of each model to determine their value for investigating pathomechanisms and testing drugs.

\section{Acknowledgments}

We thank the staff of the Biological Resources Center at the University of New South Wales for animal care.

Y.D.K. designed and supervised the study; A.v.H., G.C., J.v.d.H., M.M., S.I., L.S., M.B., P.R.A., W.S.L., M.P., and T.A.B. performed experiments; A.v.H., M.M., M.B. R.S.C., L.M.I., and Y.D.K. analyzed data; G.M.H., O.P., M.C.K., and R.S.C. acquired funding support; and L.M.I. and Y.D.K. wrote the manuscript with input from all authors.

\section{Supplemental Data}

Supplemental material for this article can be found at https://doi.org/10.1016/j.ajpath.2018.03.002.

\section{References}

1. Burrell JR, Halliday GM, Kril JJ, Ittner LM, Gotz J, Kiernan MC, Hodges JR: The frontotemporal dementia-motor neuron disease continuum. Lancet 2016, 388:919-931

2. Neumann M, Sampathu DM, Kwong LK, Truax AC, Micsenyi MC, Chou TT, Bruce J, Schuck T, Grossman M, Clark CM, McCluskey LF, Miller BL, Masliah E, Mackenzie IR, Feldman H, Feiden W, Kretzschmar HA, Trojanowski JQ, Lee VM: Ubiquitinated TDP-43 in frontotemporal lobar degeneration and amyotrophic lateral sclerosis. Science 2006, 314:130-133

3. Josephs KA, Murray ME, Whitwell JL, Parisi JE, Petrucelli L, Jack CR, Petersen RC, Dickson DW: Staging TDP-43 pathology in Alzheimer's disease. Acta Neuropathol 2014, 127:441-450

4. Buratti E, Baralle FE: Characterization and functional implications of the RNA binding properties of nuclear factor TDP-43, a novel splicing regulator of CFTR exon 9. J Biol Chem 2001, 276:36337-36343

5. Winton MJ, Igaz LM, Wong MM, Kwong LK, Trojanowski JQ, Lee VM: Disturbance of nuclear and cytoplasmic TAR DNA-binding protein (TDP-43) induces disease-like redistribution, sequestration, and aggregate formation. J Biol Chem 2008, 283:13302-13309

6. Ke YD, van Hummel A, Stevens CH, Gladbach A, Ippati S, Bi M, Lee WS, Kruger S, van der Hoven J, Volkerling A, Bongers A, Halliday G, Haass NK, Kiernan M, Delerue F, Ittner LM: Short-term suppression of A315T mutant human TDP-43 expression improves functional deficits in a novel inducible transgenic mouse model of FTLD-TDP and ALS. Acta Neuropathol 2015, 130:661-678

7. Walker AK, Spiller KJ, Ge G, Zheng A, Xu Y, Zhou M, Tripathy K, Kwong LK, Trojanowski JQ, Lee VM: Functional recovery in new mouse models of ALS/FTLD after clearance of pathological cytoplasmic TDP-43. Acta Neuropathol 2015, 130:643-660

8. Walker AK, Tripathy K, Restrepo CR, Ge G, Xu Y, Kwong LK, Trojanowski JQ, Lee VM: An insoluble frontotemporal lobar degeneration-associated TDP-43 C-terminal fragment causes neurodegeneration and hippocampus pathology in transgenic mice. Hum Mol Genet 2015, 24:7241-7254

9. Vanden Broeck L, Callaerts P, Dermaut B: TDP-43-mediated neurodegeneration: towards a loss-of-function hypothesis? Trends Mol Med 2014, 20:66-71

10. Kabashi E, Valdmanis PN, Dion P, Spiegelman D, McConkey BJ, Vande Velde C, Bouchard JP, Lacomblez L, Pochigaeva K, Salachas F, Pradat PF, Camu W, Meininger V, Dupre N, Rouleau GA: TARDBP mutations in individuals with sporadic and familial amyotrophic lateral sclerosis. Nat Genet 2008, 40:572-574

11. Van Deerlin VM, Leverenz JB, Bekris LM, Bird TD, Yuan W, Elman LB, Clay D, Wood EM, Chen-Plotkin AS, Martinez-Lage M, Steinbart E, McCluskey L, Grossman M, Neumann M, Wu IL, Yang WS, Kalb R, Galasko DR, Montine TJ, Trojanowski JQ, Lee VM, Schellenberg GD, Yu CE: TARDBP mutations in amyotrophic lateral sclerosis with TDP-43 neuropathology: a genetic and histopathological analysis. Lancet Neurol 2008, 7:409-416

12. DeJesus-Hernandez M, Mackenzie IR, Boeve BF, Boxer AL, Baker M, Rutherford NJ, Nicholson AM, Finch NA, Flynn H, Adamson J, Kouri N, Wojtas A, Sengdy P, Hsiung GY, Karydas A, Seeley WW, Josephs KA, Coppola G, Geschwind DH, Wszolek ZK, Feldman H, Knopman DS, Petersen RC, Miller BL, Dickson DW, Boylan KB, 
Graff-Radford NR, Rademakers R: Expanded GGGGCC hexanucleotide repeat in noncoding region of C9ORF72 causes chromosome 9plinked FTD and ALS. Neuron 2011, 72:245-256

13. Renton AE, Majounie E, Waite A, Simon-Sanchez J, Rollinson S, Gibbs JR, et al: A hexanucleotide repeat expansion in C9ORF72 is the cause of chromosome 9p21-linked ALS-FTD. Neuron 2011, 72: $257-268$

14. Roberson ED: Mouse models of frontotemporal dementia. Ann Neurol 2012, 72:837-849

15. Wegorzewska I, Bell S, Cairns NJ, Miller TM, Baloh RH: TDP-43 mutant transgenic mice develop features of ALS and frontotemporal lobar degeneration. Proc Natl Acad Sci U S A 2009, 106: 18809-18814

16. Wils H, Kleinberger G, Janssens J, Pereson S, Joris G, Cuijt I, Smits V, Ceuterick-de Groote C, Van Broeckhoven C, Kumar-Singh S: TDP-43 transgenic mice develop spastic paralysis and neuronal inclusions characteristic of ALS and frontotemporal lobar degeneration. Proc Natl Acad Sci U S A 2010, 107:3858-3863

17. Igaz LM, Kwong LK, Chen-Plotkin A, Winton MJ, Unger TL, Xu Y, Neumann M, Trojanowski JQ, Lee VM: Expression of TDP-43 Cterminal fragments in vitro recapitulates pathological features of TDP43 proteinopathies. J Biol Chem 2009, 284:8516-8524

18. Cannon A, Yang B, Knight J, Farnham IM, Zhang Y, Wuertzer CA, D'Alton S, Lin WL, Castanedes-Casey M, Rousseau L, Scott B, Jurasic M, Howard J, Yu X, Bailey R, Sarkisian MR, Dickson DW, Petrucelli L, Lewis J: Neuronal sensitivity to TDP-43 overexpression is dependent on timing of induction. Acta Neuropathol 2012, 123: 807-823

19. D'Alton S, Altshuler M, Cannon A, Dickson DW, Petrucelli L, Lewis J: Divergent phenotypes in mutant TDP-43 transgenic mice highlight potential confounds in TDP-43 transgenic modeling. PLoS One 2014, 9:e86513

20. Igaz LM, Kwong LK, Lee EB, Chen-Plotkin A, Swanson E, Unger T, Malunda J, Xu Y, Winton MJ, Trojanowski JQ, Lee VM: Dysregulation of the ALS-associated gene TDP-43 leads to neuronal death and degeneration in mice. J Clin Invest 2011, 121:726-738

21. Becker LA, Huang B, Bieri G, Ma R, Knowles DA, Jafar-Nejad P, Messing J, Kim HJ, Soriano A, Auburger G, Pulst SM, Taylor JP, Rigo F, Gitler AD: Therapeutic reduction of ataxin-2 extends lifespan and reduces pathology in TDP-43 mice. Nature 2017, 544:367-371

22. Wang W, Wang L, Lu J, Siedlak SL, Fujioka H, Liang J, Jiang S, Ma X, Jiang Z, da Rocha EL, Sheng M, Choi H, Lerou PH, Li H, Wang X: The inhibition of TDP-43 mitochondrial localization blocks its neuronal toxicity. Nat Med 2016, 22:869-878

23. Perera ND, Sheean RK, Crouch PJ, White AR, Horne MK, Turner BJ: Enhancing survival motor neuron expression extends lifespan and attenuates neurodegeneration in mutant TDP-43 mice. Hum Mol Genet 2016, 25:4080-4093

24. Iguchi Y, Eid L, Parent M, Soucy G, Bareil C, Riku Y, Kawai K, Takagi S, Yoshida M, Katsuno M, Sobue G, Julien JP: Exosome secretion is a key pathway for clearance of pathological TDP-43. Brain 2016, 139:3187-3201

25. Delerue F, Ittner LM: Genome editing in mice using CRISPR/Cas9: achievements and prospects. Clon Transgen 2015, 4:135

26. Delerue F, White M, Ittner LM: Inducible, tightly regulated and nonleaky neuronal gene expression in mice. Transgenic Res 2014, 23 : $225-233$

27. Ullmann JF, Watson C, Janke AL, Kurniawan ND, Reutens DC: A segmentation protocol and MRI atlas of the C57BL/6J mouse neocortex. Neuroimage 2013, 78:196-203

28. van Eersel J, Stevens CH, Przybyla M, Gladbach A, Stefanoska K, Chan CK, Ong WY, Hodges JR, Sutherland GT, Kril JJ, Abramowski D, Staufenbiel M, Halliday GM, Ittner LM: Early-onset axonal pathology in a novel P301S-Tau transgenic mouse model of frontotemporal lobar degeneration. Neuropathol Appl Neurobiol 2015, 41:906-925

29. van Hummel A, Bi M, Ippati S, van der Hoven J, Volkerling A, Lee WS, Tan DC, Bongers A, Ittner A, Ke YD, Ittner LM: No overt deficits in aged tau-deficient C57B1/6.Mapttm1(EGFP)Kit GFP knockin mice. PLoS One 2016, 11:e0163236

30. Ittner A, Chua SW, Bertz J, Volkerling A, van der Hoven J, Gladbach A, Przybyla M, Bi M, van Hummel A, Stevens CH, Ippati S, Suh LS, Macmillan A, Sutherland G, Kril JJ, Silva AP, Mackay J, Poljak A, Delerue F, Ke YD, Ittner LM: Site-specific phosphorylation of tau inhibits amyloid-beta toxicity in Alzheimer's mice. Science 2016, 354:904-908

31. Morsch M, Reddel SW, Ghazanfari N, Toyka KV, Phillips WD: Muscle specific kinase autoantibodies cause synaptic failure through progressive wastage of postsynaptic acetylcholine receptors. Exp Neurol 2012, 237:286-295

32. Moon Y, Sung J, An R, Hernandez ME, Sosnoff JJ: Gait variability in people with neurological disorders: a systematic review and metaanalysis. Hum Mov Sci 2016, 47:197-208

33. Fogarty MJ, Klenowski PM, Lee JD, Drieberg-Thompson JR, Bartlett SE, Ngo ST, Hilliard MA, Bellingham MC, Noakes PG: Cortical synaptic and dendritic spine abnormalities in a presymptomatic TDP-43 model of amyotrophic lateral sclerosis. Sci Rep 2016, 6:37968

34. Handley EE, Pitman KA, Dawkins E, Young KM, Clark RM, Jiang TC, Turner BJ, Dickson TC, Blizzard CA: Synapse dysfunction of layer $\mathrm{V}$ pyramidal neurons precedes neurodegeneration in a mouse model of TDP-43 proteinopathies. Cereb Cortex 2017, 27:3630-3647

35. Spiller KJ, Cheung CJ, Restrepo CR, Kwong LK, Stieber AM, Trojanowski JQ, Lee VM: Selective motor neuron resistance and recovery in a new inducible mouse model of TDP-43 proteinopathy. J Neurosci 2016, 36:7707-7717

36. Iancu Ferfoglia R, Heritier Barras AC, Pollak P, Janssens JP, Pradat PF, Allali G: Gait performance and use of mental imagery as a measure of disease progression in amyotrophic lateral sclerosis. Eur Neurol 2016, 75:109-112

37. Xia Y, Gao Q, Lu Y, Ye Q: A novel approach for analysis of altered gait variability in amyotrophic lateral sclerosis. Med Biol Eng Comput 2016, 54:1399-1408

38. Radovanovic S, Milicev M, Peric S, Basta I, Kostic V, Stevic Z: Gait in amyotrophic lateral sclerosis: is gait pattern differently affected in spinal and bulbar onset of the disease during dual task walking? Amyotroph Lateral Scler Frontotemporal Degener 2014, 15:488-493

39. Xu YF, Zhang YJ, Lin WL, Cao X, Stetler C, Dickson DW, Lewis J, Petrucelli L: Expression of mutant TDP-43 induces neuronal dysfunction in transgenic mice. Mol Neurodegener 2011, 6:73

40. Mancuso R, Olivan S, Osta R, Navarro X: Evolution of gait abnormalities in SOD1(G93A) transgenic mice. Brain Res 2011, 1406 $65-73$

41. Ramirez-Jarquin UN, Tapia R: Excitatory and inhibitory neuronal circuits in the spinal cord and their role in the control of motor neuron function and degeneration. ACS Chem Neurosci 2018, 9:211-216

42. Vergouts M, Marinangeli C, Ingelbrecht C, Genard G, Schakman O, Sternotte A, Calas AG, Hermans E: Early ALS-type gait abnormalities in AMP-dependent protein kinase-deficient mice suggest a role for this metabolic sensor in early stages of the disease. Metab Brain Dis 2015 , 30:1369-1377

43. Schludi MH, Becker L, Garrett L, Gendron TF, Zhou Q, Schreiber F, Popper B, Dimou L, Strom TM, Winkelmann J, von Thaden A, Rentzsch K, May S, Michaelsen M, Schwenk BM, Tan J, Schoser B, Dieterich M, Petrucelli L, Holter SM, Wurst W, Fuchs H, GailusDurner V, de Angelis MH, Klopstock T, Arzberger T, Edbauer D: Spinal poly-GA inclusions in a C9orf72 mouse model trigger motor deficits and inflammation without neuron loss. Acta Neuropathol 2017, 134:241-254 